\section{Der standardisierte Uhrentest bei Schulkindern der Klassenstufen I-IV}

Horst J. Koch ${ }^{1}$, Berno Hahn², Alexander Szecsey

${ }^{1}$ Psychiatrische Universitätsklinik Regensburg

2 Grundschule Parsberg

psychoneuro 2005; 31 (3): 154-156

Der Uhrentest hat sich in der geriatrischen Praxis als probate Untersuchung zur schnellen orientierenden Dokumentation der kognitiven Fähigkeiten von Patienten mit Hirnleistungsstörung bewährt. In der vorliegenden Arbeit wurde dieser standardisierte Test bei 105 Kindern (Alter 6-11 Jahre) der Klassen I-IV anonymisiert erhoben und von zwei unabhängigen Ratern im Abstand von vier Wochen nach der 6stufigen Shulman-Skala beurteilt. Im Uhrentest finden sich vor allem zwischen den Jahrgangsstufen I/II und III/IV Leistungszuwächse, wobei die diskriminierende Funktion des Tests maximal bis zur IV. Klassenstufe zu reichen scheint. Die Intra-Rater-Reliabilitäten lagen um 0,8, während die Inter-Rater-Reliabilitäten in Abhängigkeit vom Zeitpunkt zwischen 0,6 und 0,8 lagen (Spearman-Rangkorrelation). Mit der Kanonischen Korrelation errechneten sich eine Intra-Rater-Konsistenz von 0,93 und eine Inter-Rater-Konsistenz von 0,82.

$\mathrm{M}$ it dem Uhrentest (UT, Uhrenzeichnen, clock drawing test, clock face test) lassen sich kognitive Störungen in der Geriatrie schnell erfassen. Der praktische Vorteil dieses Instrumentariums liegt in seiner einfachen Handhabung, der Unabhängigkeit von Testmaterialien und der kurzen Testdauer von etwa fünf Minuten. Der Patient/Proband wird gebeten, zu einer vorgegebenen Zeit die Zeiger und Ziffern einer Uhr in einen Kreis einzufügen. Beurteilt werden die Güte der Zeiger und Ziffern in Bezug zur Aufgabenstellung. Von den vorgeschlagenen Rangskalen zur Quantifizierung hat sich das Verfahren nach Shulman (14) bewährt. In die Uhrentest-Aufgabe fließen sowohl visuokonstruktive und visuoperzeptive als auch mnestische Fähigkeiten ein. Der Uhrentest hat sich in der gerontopsychiatrischen Praxis als probates und für die älteren Patienten stressfreies Instrumentarium bewährt, um die kognitive Leistungsfähigkeit innerhalb weniger Minuten einzuschätzen und zu doku- mentieren (8). Zwischen den Uhrentest-Rängen und anderen Testverfahren (Syndrom-Kurztest, Mini-Mental-Status-Examination, Strukturiertes Interview zur Diagnose einer Demenz nach Zaudig) fanden Gürtler et al. Beträge des Spearman-Korrelationskoeffizienten zwischen 0,56 und 0,62. Die Korrelationen zwischen dem UT und dem SKT bzw. UT und MMSE lagen in der retrospektiven Studie von Schramm et al. (12) bei 0,60 bzw. $-0,68$. Die Inter-Rater-Reliabilität des Uhrentests wird in der Literatur in Abhängigkeit von den Auswertemethoden zwischen 0,84 und 0,97 angegeben $(10,16)$. Death et al. (7) bestimmten die Sensitivität zu 0,77 und die Spezifität zu 0,87 , wobei sie die Benutzerfreundlichkeit des Tests in nichtpsychiatrischen Kliniken betonten. Von leichten depressiven Syndromen wurde das Ergebnis des Uhrentests nicht substantiell beeinflusst (4). Die vergleichsweise guten Korrelationen der Uhrentests mit Standard-Testverfahren (MMSE, SKT) verringerten sich aber deutlich,

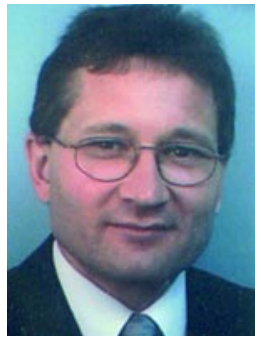

Horst J. Koch wenn nur geringe kognitive Defizite vorliegen (13). Cohen et al. (6) untersuchten erstmals sechs- bis zwölfjährige Kinder mit dem Uhrentest. Sie diskutierten die Leistungen im Uhrentest als Indikator für die Entwicklung und Reifung des Frontallappens, dem wichtige Funktionen im Hinblick auf Sprache, Motorik und Emotionen zukommt. Neben der Planung motorischer Handlungen und $\mathrm{Zu}-$ sammenfügung von Handlungsteilen ist der frontale Kortex notwendig, um automatisierte Abläufe zu initiieren und zielgerichtetes Verhalten zu erlernen $(2,3)$.

In der vorliegenden Untersuchung wurden die Leistungen im Uhrentest bei Schulkindern der Klassenstufen 1 bis 4 als Funktion der Klassenstufe dargestellt und erstmals Gütekriterien für diese Altersgruppe berechnet.

\section{Methode}

105 Schulkinder Altersstufen 1 bis 4 (m: 54, w: 51; Alter 6-11 Jahre) einer Grundschule wurden nach Genehmigung durch die Schulbehörde gebeten, eine Uhr mit der vorgegebenen Zeit „10 Minuten nach $11.00 \mathrm{Uhr}$ “ zu zeichnen. Eine wünschenswerte Wiederholung der Untersuchung war nicht möglich. Die anonymisiert erhobenen Daten wurden danach im Abstand von vier Wochen von je zwei identischen unabhängigen Ratern nach den Kriterien von Shulman et al. (14) auf einer sechsstufigen Rangskala [1 = perfekt; 2 = leichte visuell-räum- 
liche Fehler; 3 = fehlerhafte Uhrzeit bei erhaltener visuell-räumlicher Darstellung der Uhr; 4 = mittelgradige visuell-räumliche Desorganisation, sodass ein korrektes Einzeichnen der Uhrzeit unmöglich ist; 5 = schwergradige visuell-räumliche Desorganisation; 6 = keinerlei Darstellung einer Uhr] beurteilt. Beide Rater wurden unabhängig voneinander über Uhrentests ausführlich instruiert und mussten 100 geriatrische Routine-Tests anhand der Shulman-Skala bewerten.

Alle Daten wurden deskriptiv ausgewertet (Mittelwert/SD, Error bar plots, Abb. 1). Als Schätzer für die Intra- und Inter-Rater-Reliabiliät der rangskalierten Daten wurde der Spearman-Rang-Korrelationskoeffizient herangezogen $(1,15)$. Als weiterer Schätzer der Intra- und Inter-Rater-Gesamtkonsistenz wurden kanonische Korrelation (Koeffizienten $\mathrm{R}$ für Rater 1 versus Rater 2 und Zeitpunkt 1 versus Zeitpunkt 2) berechnet. Alle Berechnungen wurden mit Statistica (Version 6.0; Tulsa, USA) durchgeführt. Testergebnisse zwischen den Klassenstufen wurden mittels der nichtparametrischer Kruskal-Wallis-ANOVA für unverbundene Stichproben getrennt für jeden Rater verglichen. Als Zweistichprobentest (post hoc Lokalisationstest) wurde der Mann-Whitney-Test für unabhängige Stichproben herangezogen (15). Ein P-Wert < 0,05 wurde als signifikant angesehen.

\section{Ergebnisse}

In Abhängigkeit von der Klassenstufe nehmen die Fertigkeiten der Schüler kontinuierlich und signifikant unabhängig vom Rater zu (Abb. 2). Die P-Werte der Post-hocTests sind in der Tabelle 1 zusammengestellt; es fällt auf, dass Rater 1 deutlicher zwischen sukzessiven Klassenstufen diskriminiert. Die wichtigsten Lernschritte liegen dabei offensichtlich zwischen den Klassen I und II bzw. III und IV, während sich die Klassenstufe II und III nur unwesentlich unterscheiden. Ab der Klassenstufe IV scheint das Uhrenzeichnen keine relevante diskriminierende Funktion mehr zu haben, da im Mittel maximale Scores erzielt werden. Zwischen Mädchen und Buben findet sich kein signifikanter Un- terschied, wobei die Leistungen der Mädchen im Mittel tendenziell um 0,1 bis 0,3 Scores besser ausfallen.

Die Intra-Rater-Reliabilitäten betragen zwischen 0,77 und 0,89. Die Inter-Rater-Reliabilitäten liegen mit 0,59 bis 0,78 etwas niedriger. Die entsprechenden Spearman-Korrelationskoeffizienten als Schätzer der Reliabilitäten sind in der Tabelle 2 zusammengefasst. Als Beispiel zeigt die Abbildung 2 das Korrelationsdiagramm und die Regressionsgerade der IntraRater-Reliabilität des Raters 1. In Bezug auf die Inter-Rater-Konsistenz errechnet sich ein kanonischer Korrelationskoeffizient $\mathrm{R}$ (Rater 1 versus Rater 2 ) von 0,82 ( $p<0,05)$. Die IntraRater-Konsistenz (Zeitpunkt 1 versus Zeitpunkt 2) fällt mit 0,93 ( $p<0,05)$ geringgradig höher aus. Das kanonische Korrelationsdiagramm für die Intra-Rater-Konsistenz ist in der Abbildung 3 wiedergegeben.

\section{Diskussion}

In der vorliegenden Studie wurden erstmals intra- und interindividuelle Reliabilitäten des Uhrentests bei Schulkindern zwischen 6 und 11 Jahren berechnet. Der Uhrentest hat sich auch in dieser Altersgruppe als reliable und einfache Untersuchung erwiesen und gibt einen Hinweis auf den Entwicklungsstand des Kindes. Inter-Rater-Reliabilitäten um 0,8 stimmen mit den Werten der Literatur überein (10). Der Verlauf der Scores als Funktion der Klassenstufe deutet darauf hin, dass der Uhrentest bei normal entwickelten Kindern in einem Alter von etwa 10 Jahren keine diskriminierende Power in Bezug auf die Testleistung mehr hat. Dies entspricht dem „Ceiling effect“, wie er auch beim MMSE bei leichten kognitiven Störungen beobachtet wird (8). Weitere Untersuchungen mit Testwiederholungen und Vergleichstests sind notwendig, um die Test-RetestReliabilität zu messen und die Validität einzuschätzen.

Neben dem Frontallappen, der insbesondere für die Zielsteuerung einer Handlung wichtig ist, könnte der Parietallappen von funktioneller Bedeutung für die visuokonstruktiven (links) und visuoperzeptiven (rechts) Aufgaben des Uhrentests sein (3). Anatomische Untersuchungen deuten

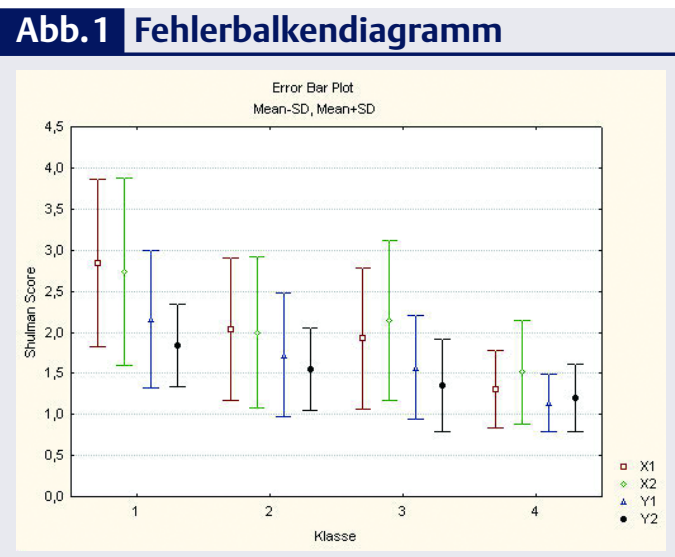

Fehlerbalkendiagramm (Mittelwert, SD) der Uhrentest-Scores als Funktion der Klassenstufe mit signifikanter Zunahme der Leistung (ANOVA, $\mathrm{p}<\mathbf{0 , 0 5}$ ). Die Werte für Rater 1 (X) und Rater 2 (Y) sind separat für jeden Zeitpunkt dargestellt ( $X 1$, $\mathrm{X} 2, \mathrm{Y} 1, \mathrm{Y} 2$ )

\section{Abb. 2 Korrelationsdiagramm}

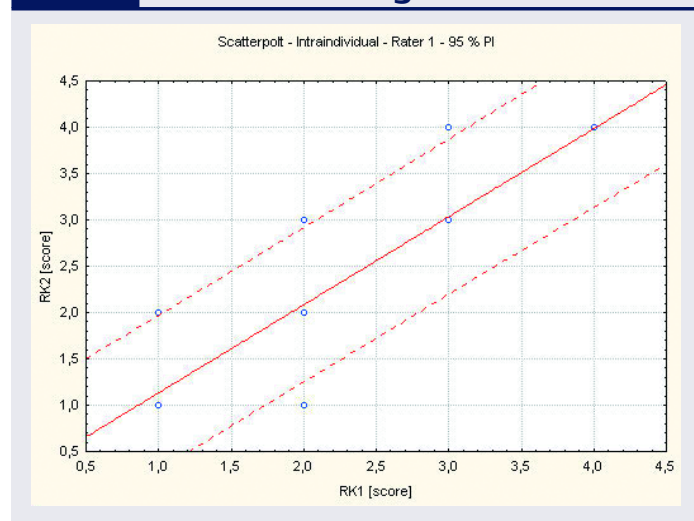

Korrelationsdiagramm zur Intra-Rater-Reliabilität (Rater 1, X) mit Regressionsgeraden $[Y=0,18+0,95$ ${ }^{*} \mathrm{X}$ ] und 95\%-Prädiktionsintervall

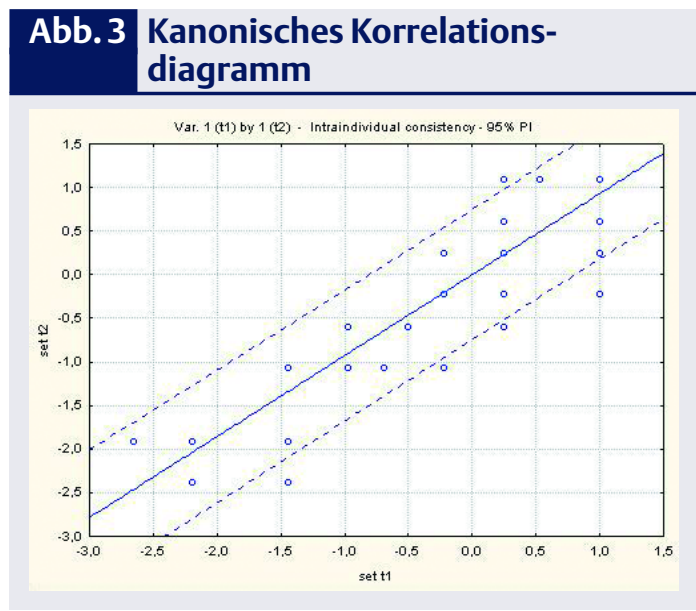

Kanonisches Korrelationsdiagramm zur IntraRater-Konsistenz mit Regressionsgeraden und 95\%-Prädiktionsintervall 
Tab. 1 P-Werte des 2-seitigen Mann-Whitney-Tests

\begin{tabular}{lllllll} 
& I-II & II-III & III-IV & I-III & I-IV & II-IV \\
\hline X, t1 & 0,008 & 0,698 & 0,010 & 0,004 & $<0,001$ & 0,002 \\
\hline X, t2 & 0,024 & 0,618 & 0,018 & 0,067 & $<0,001$ & 0,061 \\
Y, t1 & 0,100 & 0,531 & 0,015 & 0,023 & $<0,001$ & 0,004 \\
\hline Y, t2 & 0,125 & 0,174 & 0,432 & 0,008 & $<0,001$ & 0,023
\end{tabular}

P-Werte des 2-seitigen Mann-Whitney-Tests zur Lokalisation der signifikanten Differenzen zwischen den Klassenstufen 1 bis 4

darauf hin, dass vor allem das Volumen des rechten temporalen Kortex mit den Uhrentestergebnissen korreliert (5). Der temporale Kortex ist bedeutsam für Gedächtnis- und Sprachfunktionen sowie visuelle Diskriminationsleistungen. Die neuropsychologischen Defizite, wie sie bei Patienten mit rechts- oder linkshemisphärischen Läsionen, insbesondere des Parietallappens, gesehen werden, spielen bei gesunden Kindern keine Rolle. Erwachsene Patienten mit Parietallappenschädigungen haben häufig gekreuzte Defizite im Uhrenzeichnen-Test (9). Vielmehr beeinflusst der Stand des Lernens (Klassenstufe) unmittelbar das Ergebnis im Uhrentest. Die Untersuchung zeigt, dass nach der IV. Klasse die Uhr sowohl perzeptiv als auch visuokonstruktiv beherrscht wird. Die zeitlichen und räumlichen Planungsfähigkeiten und Koordination scheinen mit etwa 10 Jahren gut entwickelt zu sein, wobei insbesondere der Einfluss des Elternhauses und der Peers zur Variabilität beiträgt. Einschränkend muss ergänzt werden, dass der Test zu grob angelegt ist, um feine entwicklungsphysiologische Veränderungen des Zerebrums zu beurteilen. Die Feststellung, dass mit 10 Jahren die realistische Uhr die Regel ist, korreliert aber gut mit neuropsychologischen Erkenntnissen. Zwischen dem 7. und 9. Lebensjahr durchläuft das Kind die Periode des vorperspektivischen Zeichnens, das ab 9 bis 10 Jahren dem perspektivischen Zeichnen weicht (11). Um die

\section{Tab. 2 Spearman-Korrelationsmatrix}

\begin{tabular}{llll} 
& Rater 1,t2 & Rater $\mathbf{2 , t}$ 1 & Rater 2, t2 \\
\hline Rater 1, t1 & 0,89 & 0,81 & 0,65 \\
\hline Rater 1, t2 & 0,78 & 0,59 \\
\hline Rater 2, t1 & & 0,77 \\
& & & $(\mathrm{p}<0,05)$
\end{tabular}

kognitive Entwicklung von Kindern zwischen 6 und 10 Jahren einzuschätzen und im Verlauf zu dokumentieren, würde sich der Uhrentest auf Grund seiner einfachen Handhabung anbieten. Weitere vergleichende Untersuchungen sind aber notwendig, um seine prognostische Aussagekraft beurteilen zu können.

\section{The stardardized clock test for school} children of the $1^{\text {st }}$ to the $4^{\text {th }}$ form The clock test (clock drawing test, clock face test) has proven to be a fast instrument to assess the cognitive performance of patients with supposed dementia. This study analysed the anonymous clock test data of 105 school children of the $1^{\text {st }}$ to the $4^{\text {th }}$ form. Two independent raters evaluated the tests based on Shulman's scores (6 ranks) on two occasions observing an interval of 4 weeks. Major improvements of performance were found between the classes I/II and III/IV, respectively. The discriminant capability of the test seems to be weak at the level of the $4^{\text {th }}$ form. The intra-rater and inter-rater reliability (Spearman correlation) was found to be about 0.8 or between 0.6 and 0.8 , depending on the time of assessment. Canonical correlations as a measure of inter-rater consistency or interrater-consistency were 0.93 and 0.82 .

\section{Key Words}

Clock drawing test - school children reliability

\section{Literatur}

1. American Educational Research Association, American Psychological Association and National Council of Measurement in Education: Standards for educational and psychological testing. Washington, American Psychological Association, 1985

2. Bernstein NA. Bewegungsphysiologie. Sportmedizinische Schriftenreihe 9, Ambrosius Barth, Leipzig 1988: 120-137

3. Birbaumer N, Schmidt RF. Biologische
Psychologie: Kognitive Prozesse (Denken). Heidelberg, Springer, 1991: 620-654

4. Brodaty $\mathrm{H}$, Moore M. The clock drawing test for dementia of Alzheimer's type: A comparison of three scoring methods in a memory disorder clinic. Int J Geriatr Psychiatry 1997; 12: 619-627

5. Cahn-Weiner DA, Sullivan EV, Schear PK, Fama R, Lim KO et al. Brain structural and cognitive correlates of clock drawing performance in Alzheimer's disease. J Int Neuropsychol Soc 1999; 5: 502-509

6. Cohen MJ, Ricci CA, Kibby MY, Edmonds JE. Developmental progression of clock face drawing in children. Neuropsychol Dev Cogn Sect C Child Neuropsychol 2000; 6: 64-76

7. Death J, Douglas A, Kenny RA. Comparison of clock drawing with Mini Mental State Examination as a screening in elderly acute hospital admission. Postgrad Med J 1993; 69: 696-700

8. Gürtler K, Szecsey A, Stöhr H. Psychometrische Demenzdiagnostik in der klinischen Praxis der Gerontopsychiatrie. Z Gerontol Geriatrie 1998; 31: 281-185

9. Kaplan E. A process approach to neuropsychological assessment. In: Boll T, Bryant BK (eds.). Clinical Neuropsychology and Brain Function: Research, Measurement, and Practice. American Psychological Association, 1988

10. Lezak D. Neuropsychological Assessment: Clock Face. Oxford, Oxford University Press, 1995: 586-687

11. Schraml WJ. Einführung in die moderne Entwicklungspsychologie für Pädagogen und Sozialpädagogen: Entwicklung des Zeichnens und Malens - kreativer Aspekt. Frankfurt, Ullstein, 1980: 425-435

12. Schramm U, Berger $G$, Müller $R$ et al. Psychometric properties of clock drawing test and MMSE of short performance test (SKT) in dementia screening in a memory clinic population. Int J Geriatr Psychiatry 2002; 17: 254-260

13. Seigerschmidt E, Mösch E, Siemen M, Förstl H, Bickel H. The clock drawing test and questionable dementia: reliability and validity. Int J Geriatr Psychiatry 2002; 17 : 1048-1054

14. Shulman KI, Gold DP et al. Clock drawing and dementia in the community. A longitudinal study. Int J Geriatr Psychiatry 1993; 8: 487-496

15. Siegel S, Castellan NJ. Nonparametric statistics for the behavioral sciences. New York, McGraw-Hill, 1988: 225-244

16. Sunderland T, Hill JL, Mellow AM, Lawlor $B A$ et al. Clock drawing in Alzheimer's disease: A novel measure of dementia severity. Am Geriatr Soc 1998; 37: 725-729

\section{Korrespondenzadresse:}

Dr. med. Dr. rer. nat. Horst J. Koch MFPM DCPSA

Facharzt für Klinische Pharmakologie,

Psychiatrie und Psychotherapie

Psychiatrische Universitätsklinik

im Bezirksklinikum

Universitätsstraße 84

93053 Regensburg

horst.koch@medbo.de 